\title{
Occurrence of Left Ventricular Hypertrophy in Subjects with Metabolic Syndrome: Role of Adiponectin
}

\author{
Tiziana Di Chiara, Salvatore Corrao, Alessandro Raffa, Antonio Pinto, Rosario Scaglione*
}

Dipartimento Biomedico di Medicina Clinica e Specialistica, Università degli studi di Palermo (ltaly)

*Corresponding author: Rosario Scaglione, MD, Associate Professor of Internal Medicine, Piazza delle Cliniche 2,90127 Palermo (Italy), Tel: 91-6552155/ Fax: 91-6552175; E-mail: rosarioscaglione@yahoo.it

\begin{abstract}
This study was designed to evaluate the role of total plasma adiponectin (ADPN) on the occurrence of left ventricular hypertrophy in subjects with metabolic syndrome (MetS) compared to a control group without MetS. The main finding of our study concerns the independent predictor role of ADPN in the occurrence of LVH in all the patients studied. Accordingly, circulating ADPN might be considered a nonhaemodynamic factor of LVH in subjects with MetS.
\end{abstract}

Keywords: Adiponectin; Metabolic syndrome; Left ventricular hypertrophy
Received date: May 12, 2016

Accepted date: February 01, 2017

Published date: February 06, 2017

Citation: Scaglione, R., et al. Occurrence of Left Ventricular Hypertrophy in Subjects with Metabolic Syndrome: Role of Adiponectin. (2017) J Diab Obes 4(1): 7-10.

DOI: $10.15436 / 2376-0494.17 .920$

\section{Introduction}

Accumulation of intra-abdominal visceral fat and of adipocyte's dysfunction are considered the cellular basis of metabolic syndrome (MetS), associated with heightened risk for cardiovascular disease and cardiovascular mortality ${ }^{[1-4]}$. Population-based studies indicated an association between MetS and abnormal left ventricular (LV) geometry ${ }^{[5,6]}$. It is well known that several factors, both hemodynamic and non-hemodynamic, may be implicated in the increase of left ventricular mass (LVM) and wall thickness and thus in the occurrence of LV hypertrophy $(\mathrm{LVH})^{[7,8]}$. In this field an important role has been attributed recently to adipose tissue dysfunction ${ }^{[1-3,9]}$, a term indicating a state of hyper secretion of pro-atherogenic, pro-inflammatory and pro-diabetic adipocytokines, which is accompanied by a decreased production of adiponectin ${ }^{[9]}$. Adiponectin (ADPN) is an adipocyte-specific protein abundantly present in the plasma with well known anti-atherogenic, anti-diabetic and anti-inflammatory properties ${ }^{[1-3,10]}$.

Despite these findings, few studies addressed the influence of circulating ADPN in the development of LVH in subjects with MetS. The present study was designed to explain the interaction between ADPN and MetS on the cardiac damage.
Accordingly, cardiac parameters and prevalence of LVH and coronary artery disease (CAD) were evaluated in patients with and without MetS. The principal objective of our study was to demonstrate that hypoadiponectinemia might be considered a nonhaemodynamic factor in the occurrence of LVH in subjects with MetS.

\section{Methods and Materials}

\section{Subjetes}

In this cross-sectional study, 205 consecutive outpatients referred to our center of hypertension and metabolic disease at the University of Palermo (Italy) from January 2013 to April 2014 because of BP or metabolic control related problems were evaluated. The study population consisted of 79 patients affected by MetS, according to NCEP/ATPIII criteria ${ }^{[1-4]}$, and 126 without MetS, utilized as controls.

A written informed consent was collected from all the subjects recruited. The protocol of this study was approved by the board of University of Palermo (Italy). Clinic examination including medical history, measurements of body weight, 
height, blood pressure (BP) and resting electrocardiogram was assessed in all the subjects. Information regarding medical history, drug use, and alcohol and cigarette consumption was collected. Exclusion criteria included alcoholism and psychiatric problems. Body mass index was calculated as weight divided by squared height and expressed as $\mathrm{kg} / \mathrm{m}^{2}$ and WHR by waist/ hip ratio ${ }^{[4]}$. Blood pressure (BP) was measured according to current recommendations with an appropriate large cuff in obese subjects. Systolic (SBP), diastolic (DBP) and mean (MBP) BP were determined. MBP was calculated by the sum of DBP plus one third of pulse pressure. According to ADA guidelines, the presence of diabetes was recognized from a fasting glycaemia $\geq$ $126 \mathrm{mg} / \mathrm{dl}$ or the use of antidiabetic drugs or insulin ${ }^{[12]}$. Insulin resistance (IR) was recognized by the evaluation of homeostasis model assessment (HOMA) index.

In all the subjects the occurrence of LVH and the prevalence of coronary artery disease were evaluated. According to echocardiographic measurement, all the subjects with LVM normalized for height to the 2.7 power (LVMI) $\geq 50 \mathrm{~g} / \mathrm{m} 2.7$ for men and $\geq 47 \mathrm{~g} / \mathrm{m} 2.7$ for women were considered to have $\mathrm{LVH}^{[13]}$.

According to guidelines of European Society of Cardiology ${ }^{[13]}$ the diagnosis of CAD was supported by history, symptoms of typical angina, cardiac markers and specific cardiac examinations.

\section{Methods}

Routine biochemical parameters were detected through standardized methods. An immune radiometric assay (Biosource, Belgium) was used to measure serum insulin. The HOMA-IR was calculated using the following equation: HOMA-IR $=$ fasting insulin $(\mathrm{pmol} / \mathrm{L} \mathrm{X}$ fasting glucose $(\mathrm{mmol} / \mathrm{L}) / 22.5$. IR was defined as an HOMA-IR in the highest quintile of its distribution (12). Adiponectin levels were measured by a validated competitive radioimmunoassay (Linco Research Inc., MO, USA) as previously described ${ }^{[4]}$.

Measurements of left ventricular geometry and function were assessed by $\mathrm{M}$ and $\mathrm{B}$-mode computerized echocardiography (ESAOTE, Italy). Accordingly, left ventricular internal diameter/height $(\mathrm{LVID} / \mathrm{h})$, interventricular septum thickness (IVST), LVM normalized for height to the 2.7 power (LVMI) (14) was calculated. Recognition of LVH by LVM/h 2.7 cut-offs has to be preferred since body surface area correction under estimates LVM in the upper range of the body surface area distribu$\operatorname{tion}^{[14]}$. The relative wall thickness (RWT) by formula [(PWTd/ LVIDd)x2] and left ventricular ejection fraction (LVEF) were also calculated with standardized method (4). Diastolic parameters, such as E/A ratio, isovolumic relaxation time (IVRT) and the deceleration time of E velocity (DTE) by echo-dopler examination were also calculated.

\section{Statistical analysis}

Data are presented as mean $\pm \mathrm{SD}$. Comparison between the groups were analyzed through the Man-Whitney U test was and comparisons between two proportions by $\mathrm{Z}$ test. Linear and multiple regression analysis were performed to study relationship among independent variables and LVMI. A p value $<0.05$ was used as cut-off for statistical significance. STAT/SE, version 9.2 for Windows (StataCorp. College Station, Texas), was used to analyze the data.

\section{Results}

Clinical characteristics, echocardiographic parameters and prevalence of $\mathrm{LVH}$ and CAD are available in Table 1. Both groups were matched with regard to sex and age. BMI ( $p<$ $0.003)$, WHR $(\mathrm{p}<0.02)$, MBP $(\mathrm{p}<0.03)$ LVM, LVMI and IVST $(\mathrm{p}<0.001)$ were significantly higher and ADPN $(\mathrm{p}<0.001)$ was significantly lower in subjects with MetS than controls. In addition, prevalence of LVH $(p<0.002)$ and CAD $(p<0.004)$ were greater in subjects with MetS than controls (Table 1).

Table 1: Clinical characteristics, metabolic profile, echocardiographic parameters and prevalence of LVH and CAD in patients with and without metabolic syndrome (MetS).

\begin{tabular}{|c|c|c|c|}
\hline & MetS & No MetS & $\mathbf{p}<$ \\
\hline Cases n. & 79 & 126 & \\
\hline $\operatorname{Sex}(M / F)$ & $17 / 26$ & $47 / 35$ & \\
\hline Age (yr) & $50.2 \pm 8.1$ & $51.0 \pm 9.3$ & n.s. * \\
\hline $\operatorname{ADPN}(\mu \mathrm{g} / \mathrm{ml})$ & $5.6 \pm 0.9$ & $9.1 \pm 2.2$ & $0.001 *$ \\
\hline BMI $\left(\mathrm{Kg} / \mathrm{m}^{2}\right)$ & $30.2 \pm 3.4$ & $28.0 \pm 5.5$ & $0.003 *$ \\
\hline WHR & $0.93 \pm 0.08$ & $0.90 \pm 0.08$ & $0.002 *$ \\
\hline MBP(mmHg) & $108 \pm 10$ & $105 \pm 8.2$ & $0.003 *$ \\
\hline Tryglycerides (mg/dl) & $124 \pm 71$ & $110 \pm 55.2$ & n.s. $*$ \\
\hline HDL-C (mg/dl) & $47.2 \pm 9.9$ & $48.1 \pm 10$ & n.s. ${ }^{*}$ \\
\hline TC (mg/dl) & $190 \pm 35.1$ & $182 \pm 34.3$ & n.s.* \\
\hline LDL-C (mg/dl) & $111.3 \pm 37.1$ & $110.2 \pm 35.3$ & n.s. ${ }^{*}$ \\
\hline HOMA-IR & $2.8 \pm 1.6$ & $2.6 \pm 1.3$ & n.s. $*$ \\
\hline LVM (gr) & $180 \pm 37$ & $152 . \pm 25.6$ & $0.001 *$ \\
\hline LVMI (gr/ h2.7) & $48 . \pm 8.1$ & $39.2 \pm 67.6$ & $0.001 *$ \\
\hline LVID/h (mm/h) & $2.90 \pm 0.19$ & $2.82 \pm 0.20$ & n.s. $*$ \\
\hline IVST (mm) & $10.5 \pm 1.6$ & $9.2 \pm 1.8$ & $0.001 *$ \\
\hline RWT & $0.42 \pm 0.07$ & $0.40 \pm 0.05$ & n.s. * \\
\hline LVEF (\%) & $63.8 \pm 2.3$ & $64.1 \pm 1.7$ & n.s. * \\
\hline $\mathbf{E} / \mathbf{A}$ & $1.5 \pm 0.5$ & $1.5 \pm 0.6$ & n.s. * \\
\hline DTE (ms) & $192 \pm 35$ & $191 \pm 30$ & n.s. * \\
\hline IVRT (ms) & $79 \pm 10$ & $82 \pm 12$ & n.s. * \\
\hline LVH pts (\%) & $43.4(23 / 53)$ & $21(17 / 82)$ & $0.002 * *$ \\
\hline CAD pts (\%) & $30.1(16 / 53)$ & $12.2(10 / 82)$ & $0.004 * *$ \\
\hline
\end{tabular}

$\mathrm{ADPN}=$ total plasma adiponectin; $\mathrm{BMI}=$ body mass index; WHR $=$ waist to hip ratio; $\mathrm{MBP}=$ mean blood pressure; HDL-C $=$ high density lipoprotein cholesterol; $\mathrm{TC}=$ total cholesterol; $\mathrm{LDL}=$ low density lipoprotein cholesterol; HOMA-IR = homeostasis model assessment of insulin resistance; $L V M=$ Left ventricular mass; LVMI = left ventricular mass/height ${ }^{2.7} ; \mathrm{LVID} / \mathrm{h}=$ left ventricular internal diameter/ height; IVST = Interventricular septal thcikness; RWT = Relative wall thickness; LVEF = Left ventricular ejection fraction; E/A: Peak early transmitral flow/peak late transmitral flow; DTE: E deceleration time; IVRT: Isovolumic relaxation time; pts = patients; $\mathrm{LVH}=$ Left ventricular hypertrophy; $\mathrm{CAD}=$ Coronary artery disease.

*ANOVA

**Z Test:

LVMI correlated directly with BMI $(\mathrm{r}=0.33 ; \mathrm{p}<$ $0.001), \operatorname{MBP}(\mathrm{r}=0.30 ; \mathrm{p}<0.001)$, MetS $(\mathrm{r}=0.28 \mathrm{p}<0.001)$ and inversely with ADPN $(\mathrm{r}=-0.55 ; \mathrm{p}<0.0001)$. Multiple regression analysis was used to explain the interaction between ADPN and MetS and to test the independent role of risk factors 
for LVMI. ADPN and BMI resulted independently associated with LVMI also when single components of MetS were includes in the analysis. (Table 2).

Table 2: Independent risk factors for LVMI assessed by multiple regression analysis.

\begin{tabular}{|l|c|c|}
\hline LVMI & $\boldsymbol{\beta} \pm$ SE & $\mathbf{P}=$ \\
\hline Age & $0.22 \pm 0.075$ & 0.004 \\
\hline Gender & $0.88 \pm 1.3$ & 0.511 \\
\hline BMI & $0.59 \pm 0.2$ & 0.005 \\
\hline ADPN & $-16.0 \pm 1.4$ & $<0.001$ \\
\hline MBP & $0.15 \pm 0.01$ & 0.167 \\
\hline HDL-C & $0.20 \pm 0.02$ & 0.205 \\
\hline Tryglycerides & $0.24 \pm 0.01$ & 0.266 \\
\hline Diabetes & $0.10 \pm 1.7$ & 0.951 \\
\hline constant & $26.4 \pm 4.1$ & $<0.001$ \\
\hline
\end{tabular}

LVMI $=$ left ventricular mass $/$ height $^{2.7} ; \mathrm{ADPN}=$ Total plasma adiponectin; $\mathrm{BMI}=$ body mass index; $\mathrm{MBP}=$ mean blood pressure; HDL-C = high density lipoprotein cholesterol

\section{Discussion}

Our data indicate clearly that subjects with MetS were characterized by decreased ADPN, increased LVMI and higher prevalence of LVH and CAD respect to controls without MetS. The main finding of the study is the independent inverse relationship between ADPN and LVMI found by us in subjects with MetS.

It is well known that MetS is often associated with LVH even if its role is partly independent from the effect of several determinants of LVM and it was not able to predict LVH independently of increased adiposity ${ }^{[15]}$. This finding is further supported by the independent LVMI/BMI relationship reported in this study. In addition circulating ADPN remained inversely and independently associated with LVMI, also when the single components of MetS were included in the analysis. To our knowledge, this is the first study to examine the role of ADPN on the changes in left ventricular geometry and on the prevalence of LVH and CAD in subjects with MetS. Our data are consistent with the suggestion that ADPN might be considered as an important nonhaemodynamic factor able to explain the occurrence of LVH in subjects at higher cardiometabolic risk ${ }^{[1-4,10]}$. These data suggest that increased risk of LVH due to a presence of MetS seems mediated mainly by adiposity and circulating ADPN which work as non hemodynamic factors. Hypoadiponectinemia or functional ADPN resistance, perhaps secondary to down regulation of ADPN receptors, may contribute to an exaggerated hypertrophic response to hemodynamic load and to inappropriate $\mathrm{LVH}^{[1,2,16]}$.

Some limitations of this study must be taken into account. First, our population is made exclusively of patients studied in the Centre of Hypertension and Metabolic Disease. This selection "bias" can explain the prevalence of MetS, diabetes, hypertension and dyslipidemia. Thus, our study is relevant for an important condition commonly found in "real life" medical practice. Second, we were not able to take into account all the details of all the different classes and subtypes of drugs assumed by the patients, which in turn might have affected the results obtained. However, this is a common limitation of many pub- lished works when "real practice" patients are studied. Third, it has been designed to be a cross-sectional study. Evaluation of the cause-effect relationship between ADPN and LVH would require a prospective study design with a cohort base and larger casistics. Therefore, we cannot prove causality or predictive ability, but only discern association.

\section{Conclusion}

In conclusion, in our patients with MetS, an increased prevalence of LVH and CAD has been found. Increasing number of MetS components was associated to step-wise increased in LVMI. ADPN and BMI separately and additively contribute to LVH in these subjects. Accordingly weight loss and increased ADPN levels may be useful to regress LVH. According to emerging data indicating that ADPN has to be considered as an important modulator of the adipovascular axis that affects the cardiometabolic risk profile, from the premetabolic syndrome, through MetS to overt atherosclerosis and that hypoadiponectinemia alone may represent an early phenomenon that long precedes the occurrence of all components of overt Met ${ }^{[1,3,17]}$, circulating ADPN might become a new target in the management and treatment of metabolic syndrome and it should be included among the criteria utilized to define MetS.

Funding: This study was in part supported by a grant of University of Palermo-Italy (2012-ATE-0278).

Disclosure: None of the Authors have any potential conflict of interest associated with this research.

Conflict of interest: All Authors state that there are not conflicts of interest related to the present paper.

\section{References}

1. Di Chiara, T., Argano, C., Corrao, S., et al. Hypoadiponectinemia: a link between visceral obesity and metabolic syndrome. (2012) J Nutr Metab: 1-7.

2. Funahashi, T. Adiponectin and the cardio metabolic syndrome: An epidemiological perspective. (2014) Best Pract Res Clinic Endocrinol Metab 28(1): 93-106.

3. Alfadda, A.A. Circulating adipokines in healthy versus unhealthy overweight and obese subjects. (2014) Int J Endocrinol: 1-7.

4. Di Chiara., T. Licata, A., Argano, C., et al. Plasma Adiponectin: A contributing factor for cardiac changes in visceral obesity-associated hypertension. (2014) Blood Press 23(3):147-153.

5. Apridonidze, T., Shaqra, H., Ktaich, N., et al. Relation of components of the metabolic syndrome to left ventricular geometry in Hispanic and non-Hispanic black adults. (2011) Am J Cardiovasc Dis 1 (1): 84-91.

6. Dharmendra Kumar, A.P., Srinivasan, S.R., Chen, W., et al. Influence of the metabolic syndrome versus the sum of its individual components on left ventricular geometry in young adults (from the Bogalusa Heart Study). (2009) Am J Cardiol 104(1): 69-73.

7. Devereux, R.B., Roman, M.J., de Simone, G., et al. Relations of left ventricular mass to demographic and hemodynamic variables in American Indians: the Strong Heart Study. (1997) Circulation 96(5): 14161423 .

8. Heckbert, S.R., Post, W., Pearson, G.D., et al. Traditional cardiovascular risk factors in relation to left ventricular mass, volume, and systolic function by cardiac magnetic resonance imaging: the Multiethnic Study of Atherosclerosis. (2006) J Am Coll Cardiol 48(11): 2285-2292. 9. Hajer, G.R., van Haeften, T.W., Visseren, F.L. Adipose tissue dys- 
function in obesity, diabetes, and vascular diseases. (2008) Eur Heart J 29(24): 2959-2971.

10. Kozakova, M., Muscelli, E., Flyvbjerg, A., et al. Adiponectin and left ventricular structure and function in healthy adults. (2008) J Clin Endocrinol Metab 93(7): 2811-2818.

11. Expert panel on detection, evaluation and treatment of high blood cholesterol in adults' Executive summary of the third report of the national cholesterol program (NCEP-ATP III). (2001) JAMA 285 (19): 2486-2497.

12. American Diabetic Association. Diagnosis and classification of diabetes mellitus. (2010) Diab Care 33 (suppl.1): S62-S69.

13. De Simone, G., Devereux, R.B., Daniels, S.R., et al. Effect of growth on variability of left ventricular mass; assessment of allometric signals in adults and children and their capacity to predict cardiovascular risk. (1995) J Am Coll Cardiol 25(5): 1056-1062.

14. Taylor, J. New ESC guidelines published on stable coronary artery disease. (2013) Eur Heart J 34(38): 2927-2930.

15. Guerra, F., Mancinelli, L., Angelini, L., et al. The association of lleft ventricular hypertrophy with metabolic syndrome is dependent on body mass index in hypertensive overweight or obese patients. (2011) Plos One 6 (1).

16. Shibata, R., Ouchi, N., Ito, M., et al. Adiponectin-mediated modulation of hypertrophic signals in the heart. (2004) Nat Med 10(12):13841389.

17. Mather, K.J., Goldberg, R.B. Clinical use of adiponectin as a marker of metabolic dysregulation. (2014) Best Pract Clin Endocrinol Metab 28: 107-117. 